\title{
BMJ Open Elicitation of Norwegian EQ-5D-5L values for hypothetical and experience- based health states based on the EuroQol Valuation Technology (EQ-VT) protocol
}

\author{
Tonya Moen Hansen (D , ${ }^{1}$ Ylva Helland, ${ }^{1}$ Liv Ariane Augestad, ${ }^{2}$ Kim Rand, ${ }^{3}$ \\ Knut Stavem, ${ }^{4,5}$ Andrew Garratt ${ }^{1}$
}

To cite: Hansen TM, Helland $Y$ Augestad LA, et al. Elicitation of Norwegian EQ-5D-5L values for hypothetical and experience-based health states based on the EuroQol Valuation Technology (EQVT) protocol. BMJ Open 2020;10:e034683. doi:10.1136/ bmjopen-2019-034683

- Prepublication history for this paper is available online. To view these files, please visit the journal online (http://dx.doi. org/10.1136/bmjopen-2019034683).

Received 01 October 2019 Revised 13 May 2020 Accepted 18 May 2020

Check for updates

(C) Author(s) (or their employer(s)) 2020. Re-use permitted under CC BY-NC. No commercial re-use. See rights and permissions. Published by BMJ.

${ }^{1}$ Health Services Research, Norwegian Institute of Public Health, Oslo, Norway

${ }^{2}$ Health Management and Health Economics, University of Oslo Faculty of Medicine, Oslo, Norway

${ }^{3}$ Health Services Research Unit, Akershus University Hospital, Lorenskog, Norway

${ }^{4}$ Institute of Clinical Medicine, University of 0slo, Oslo, Norway ${ }^{5}$ Pulmonary Medicine, Akershus University Hospital, Lorenskog, Norway

Correspondence to

Tonya Moen Hansen;

TonyaMoen.Hansen@fhi.no

\section{ABSTRACT}

Introduction Norway is one of several European countries that lacks a national value set and scoring algorithm for the EuroQol five dimensions (EQ-5D). Recent studies have found differences between countries in terms of health values or preferences for health states described by instruments such as the EQ-5D. The project aims to model a national value set for the five level version of the EQ-5D based on values elicited from a representative sample of the Norwegian adult general population in terms of region, age, sex and level of education. Using a sampling strategy supporting the collection of values for both hypothetical and experienced health states, the study will have the additional aim of assessing the feasibility of collecting experience-based values in accordance with the latest $E Q$ $5 \mathrm{D}$ valuation study protocol, and comparing values with those given for hypothetical health states.

Methods and analysis Multistage random sampling and quota-sampling will contribute to representativeness. To increase the number of valuations of experienced health states, those with less than perfect health will be oversampled, increasing the total number of interviews from 1000 to $1300-1500$. The most recent $E Q-5 D$ valuation protocol will be followed which includes computer assisted face-to-face, one-to-one interviews and use of composite time trade-off and discrete choice experiments.

Ethics and dissemination The study has been reviewed and found to be outside of the scope of the ethics committee and thus not in need of ethical approval. The study findings will be disseminated through peer-reviewed publications, conference presentations and summaries for key stakeholders and partners in the field. The scoring algorithms will be available for widely used statistical software.

\section{INTRODUCTION}

Economic evaluation undertaken by the Norwegian Institute of Public Health (NIPH) and the Norwegian Medicine Agency increasingly informs decisions about the introduction of new drugs and other health technologies in Norway. ${ }^{12}$ The Norheim Committee ${ }^{3}$ and Magnussen Working Group ${ }^{4}$ proposed
Strengths and limitations of this study

This is the first Norwegian valuation study with composite time trade-off and discrete choice experiment undertaken on a scale large enough to meet the recommendations of the most recent EuroQol (EQ) five dimensions protocol.

- Sampling strategy designed to both ensure representativeness of the final sample according to geographical region, age, sex and educational level and increase the number of experience-based valuations.

- Data collection restricted to EQ protocol, primarily developed for hypothetical health state valuation, but with the additional aim of collecting experiencebased valuations. Study design does not allow for the assessment of methods other than those described in the EQ-valuation technology protocol.

- Restricted samples for comparisons of experiencebased valuations.

- High respondent burden experienced in interviews limits the scope for addressing additional methodological questions.

methods to enhance the quality of economic evaluation, thereby further strengthening the role of economic evaluation in decisionmaking. The Ministry of Health followed up these proposals in a 2016 White Paper to Parliament on principles for priority setting in healthcare. ${ }^{5}$

Given the important role and impact of economic evaluation, it is important that the methods it incorporates, including costutility analysis, are consistent with societal values regarding publicly financed healthcare. Economic evaluation, when taking into account societal values, often takes the form of cost-utility analyses with the estimation of the incremental cost per quality-adjusted life year (QALY) gained. ${ }^{6}$ QALY takes the integral of health-related quality of life (HRQoL) over time, with HRQoL represented on a 
scale where 1 indicates a preference equal to that for full health and 0 implies a health state equal to that of being dead. Values are typically derived using general population surveys where respondents consider the relative undesirability of different health states described using instruments such as the EuroQol five dimensions (EQ5D). ${ }^{7}$ After assigning values to health states described by an instrument, QALYs are calculated by multiplying the health state value by the length of time spent in each. Evaluation of alternative technologies then involves comparison of incremental QALYs gained over incremental costs for new vs existing technologies.

Several instruments are available to calculate QALYs, of which the EQ-5D is by far the most widely applied both internationally and in Norway. ${ }^{8-10}$ The EQ- $5 \mathrm{D}$, a trade mark of the EuroQol Research Foundation, is available in over 150 languages ${ }^{11}$ in the self-complete paper version, ${ }^{12}$ and national value sets and normative data exist for over 20 countries. ${ }^{73-25}$ It is brief, widely tested, and includes five important aspects of health (mobility, self-care, usual activities, pain/discomfort and anxiety/depression), with the most recent version having five levels $(5 \mathrm{~L})$ from no problems to severe problems. The EQ-5D is considered highly acceptable to most patient groups and feasible for application where a short-form general measure of health is required. The instrument has had widespread application in research including clinical trials, population health surveys, in both Norwegian ${ }^{26}$ and Swedish National Quality Registries, ${ }^{27}$ and more recently as a healthcare quality indicator as part of the National Health Service for England and Wales Patient-Reported Outcomes Measures programme. ${ }^{14}$

The Norwegian Medicines Agency recommends the use of EQ-5D in all technology assessments and the use of a $5 \mathrm{~L}$ tariff for studies where the $5 \mathrm{~L}$ version has been used. ${ }^{2}$ In the absence of a Norwegian tariff, the 2018 Agency guidelines currently recommend the use of the EQ-5D-5L tariff for England ${ }^{14}$ where EQ-5D-5L has been used. However, criticism has been levelled at the English tariff including concerns with data quality in which serious deficiencies were revealed. ${ }^{28}$ The English 5L tariff followed an early protocol, which has since been updated with the aim of improving data quality and interview techniques. Following these concerns, and in contrast to recommendations of the Norwegian Medicines Agency, National Institute for Health and Care Excellence continues to recommend the use of the $3 \mathrm{~L}$ tariff over the $5 \mathrm{~L}$ tariff, with $5 \mathrm{~L}$ values mapped onto $3 \mathrm{~L}$ where appropriate. ${ }^{29}$

The EQ-5D is widely used in Norway, including the national quality registers where it is the most widely used patient-reported outcome measure. A national EQ-5D-5L value set and scoring algorithm is highly anticipated and will enhance the validity of economic evaluations in Norway. Norwegian EQ-5D users have largely relied on the EQ-5D-3L scoring algorithm from the UK, ${ }^{30}$ with a crosswalk-based approach $^{31}$ for studies that have used the $5 \mathrm{~L}$ version. Crosswalk-based approaches have several limitations related to issues with data dependency and differences in scale range, and are an interim solution pending a national $5 \mathrm{~L}$ value set. $^{31-33}$ Cross-national comparisons of national EQ-5D-5L value sets also suggest that there might be substantial differences across countries ${ }^{134}$ with culture and values having a role. ${ }^{35}$ Values for health for the $5 \mathrm{~L}$ version of the EQ-5D, that are representative for the Norwegian general population, will enhance the validity and legitimacy of economic evaluation in Norway.

With few exceptions, ${ }^{36-38}$ existing EQ-5D value sets are based on the general population valuing hypothetical health states, which follows recommendations that economic evaluation should include societal preferences. ${ }^{39}$ In recent years, there has been some criticism levelled at this approach, questioning the validity of health state valuations from a general population lacking the adequate experience or knowledge of the health states which they value in the form of hypothetical health states. ${ }^{40}{ }^{41}$ An alternative approach, as recommended by Sweden's Dental and Pharmaceutical Benefits Agency, ${ }^{42}$ involves individuals valuing their own health state to give experience-based values or basing their valuations on other forms of experience. The debate on whether to use hypothetical or experience-based values is to a certain extent a normative issue, relating to what we aim to maximise. ${ }^{43}$ However, there are a number of empirical questions pertaining to experience-based values. Arguably, patients have a better understanding of the consequences of reduced health on quality of life. ${ }^{414-46}$ On the other hand, they may have trouble imagining life in full health, may under-report impact of disease due to adaptation or changes in expectations over time, ${ }^{447}$ or may be less inclined to value their current health state as a state that is worse than being dead. Experience-based valuations, if better understood and elicited from representative samples of the general population may, however, be suitable for inclusion as societal values. The feasibility of collecting experience-based values, the assessment of how those with less than perfect health value their current health state and other health states in general, and how different forms of experience may influence health state valuations, are new areas for research to which this study will contribute. ${ }^{48}$

The project will derive a Norwegian EQ-5D-5L value set representative of region, age, sex and level of education composition in the Norwegian adult general population. Furthermore, the study will allow for comparisons of experience-based and hypothetical health state valuation.

\section{METHODS AND ANALYSIS}

Values for EQ-5D-5L health states will be obtained by electronic data collection including computer assisted faceto-face, one-to-one interviews and the use of composite time trade-off (cTTO) and discrete choice experiments (DCE) ${ }^{49}{ }^{50}$ The latest EQ-5D-5L protocol will be followed including EQ-valuation technology (EQ-VT). 


\section{Sampling}

Respondents must be aged 18 years or older, resident in Norway and proficient in Norwegian. Following EQ-VT protocol, sample size is set to a minimum of 1000 individuals with each valuing 10 health states, which gives the recommended 10000 responses. ${ }^{50}$ Additional 300-500 interviews, based on the oversampling of those with less than perfect health, will increase the number of valuations of experienced health states.

Norway is a Northern European country with a population of slightly more than 5 million, and a universal healthcare system. The population covers a comparatively large land mass, and for many there may be several hours travel time to the nearest hospital or large city. Urbanisation has further contributed to variation in demographic characteristics at the regional level. These factors combined with local culture, politics and traditions mean that geographical considerations are important to the design of the study.

The study will use a combination of multistage random sampling and quota sampling ensuring representativeness according to geography, age, sex and educational level. The first stage of sampling will be of geographical areas, here defined as municipalities within each acute care hospital catchment area. Norway's four regional health authorities include Northern, Central, Western and South-Eastern, with more than half the population residing in the South-Eastern health region. The catchment areas served by the 54 acute care hospitals cover all Norwegian residents (see figure 1). They vary considerably in the number of residents that they serve, from 15000 to 500000 residents. One acute care hospital will be randomly selected from each health region with the exception of the South-Eastern region, where three will be randomly selected to account for the disproportionate number of people residing in this region. Hospital catchment areas within each region will be sampled with proportional allocation, ensuring equal probability proportionate to the number of people residing in each area within the region.

Within each sampled geographical area, the possible locations for data collection will constitute the sample frame for the second stage of sampling (table 1). Locations will include public places (eg, public libraries, town halls), workplaces, recreational organisations (eg, sports clubs) and healthcare providers (eg, hospitals, rehabilitation institutions). The bodies concerned must be willing to grant the study permission for data collection and cooperate with provision of a suitable space for completion of the interviews. The locations will act as clusters of possible respondents, stratified into groups based on the characteristics of target respondents, for example, age and educational level. Stratification will increase homogeneity per cluster and ensure the representation of specific groups less likely to participate including those with poorer health, lower socioeconomic status, or faced with time constraints, including those with young children or in full-time employment. ${ }^{51}$ Locations within each

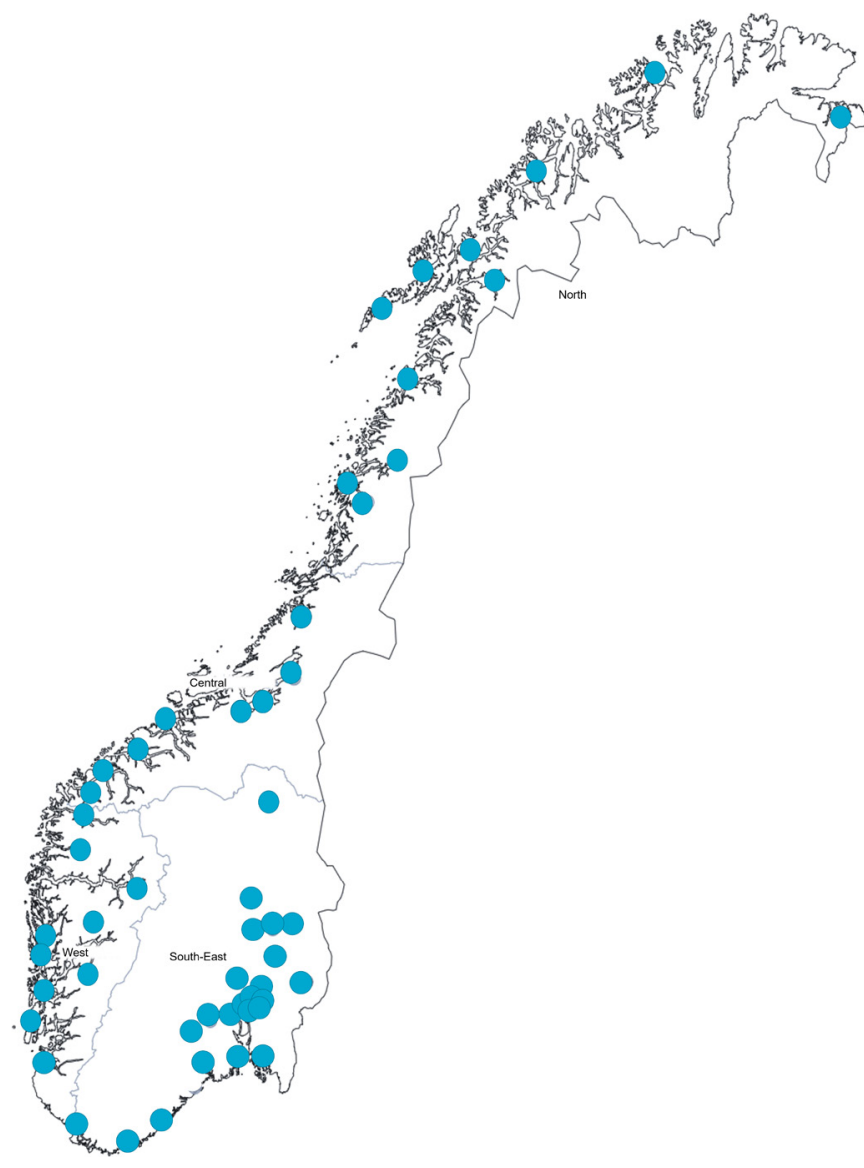

Figure 1 Hospitals with acute care function in Norway.

group in the sample frame will be randomly selected. The number of locations selected within each sample frame will be based on the size of the area and quotas. Response rates, recruitment and data quality will be assessed for the different location strata and compared across catchment areas.

Within each catchment area and at the respondent level, quota sampling will be applied according to age, sex and level of education (see tables 2 and 3). The total quota will first be allocated to each region proportionate to the number of people residing in each region. For the three regions with one sampled hospital catchment area, the quota for each of these hospital catchment areas will correspond to the regional quota. In the South-Eastern region, the regional quota is further allocated to each hospital catchment area proportionate to the number of people residing in each of these areas. The quota is then allocated to groups according to gender, age group (young adults: age 18-34, middle-aged adults: age 35-64, elderly: age 65+) and level of education (lower education, no higher than high school education, higher educationbachelor, masters or $\mathrm{PhD}$ ) equivalent to the distribution of these attributes in the respective regions. The quotas for each group are calculated using data available from http://microdata.no (see table 4), a national platform in Norway giving researchers direct access to national registries for which Statistics Norway has processing authority, such as the Norwegian National Registry, National 
Table 1 Locations for recruitment of participants, by age group and health status

\begin{tabular}{lllll}
\hline Healthy & & & & Reduced health \\
\hline Young & Middle aged & Elderly & All ages & All ages \\
\hline Places of higher education & Workplaces & Eldery homes & Public library & Hospitals \\
Child daycare facilities/ & $\begin{array}{l}\text { Recreational organisations } \\
\text { (sports teams) }\end{array}$ & $\begin{array}{l}\text { Recreational organisations } \\
\text { (choirs/orchestras) }\end{array}$ & Town hall & Rehabilitation \\
Srimary schools & Social welfare* & Community volunteer centres & centres & Health centres \\
& Adult education* & &
\end{tabular}

*Locations chosen to increase participation of those with lower socioeconomic status.

Education Database, labour market data, register for Personal Tax Payers and FD-Trygd (event history database)..$^{52}$

The study will largely rely on recruitment of potential participants by contact persons at each sampled location. Contact persons will assist in identifying and recruiting potential respondents to the study. Prior to data collection, contact persons will receive information and materials for publication in local newspapers and social media designed to enhance participation. In addition to the recruitment of respondents through locations, potential respondents will be able to contact the project group for more information about the study and enquire about participation. Potential respondents will be informed of a gift incentive. Cash has been found to be more effective than other incentives for increasing response rates and following the interview, respondents will receive a cash card equivalent to $€ 30$.

Data collection will take place from November 2019 to June 2020. Depending on the final sampling, and with an estimate of a minimum of four interviews per interviewer per day, a minimum of 55-80 working days are required for data collection. The recruitment strategy will be piloted in the catchment area sampled closest to Oslo. Necessary adjustments will follow before data collection proceeds in the rest of the country.

\section{Interviewer training}

Interviewers with Masters education level or equivalent will receive training in accordance with EuroQol Foundation guidelines and recommendations, with initial training prior to, and revised training after, the first phase of data collection. ${ }^{53}$ Based on existing studies and recommendations from EQ (Elly Stolk, personal communication), eight to twelve interviewers are required.

Quality control (QC) reports will help monitor progress and data quality throughout. ${ }^{5455}$ The reports will include assessments of protocol compliance, face validity of data collected and value distributions per interviewer. QC reports have been found to further the homogeneity of interviewer performance and reduce protocol violations and the number of inconsistent responses. ${ }^{54}$ Interviewers not meeting predefined standards will be flagged, recommended for retraining and ultimately excluded. Evaluation of the data collected and interviewer performance will be regularly discussed with interviewers in face-to-face group meetings throughout data collection, and with EQ contact persons.

\section{EuroQol valuation technology}

EQ-VT was developed to meet the challenges involved with valuation of the $5 \mathrm{~L}$ version of the $\mathrm{EQ}-5 \mathrm{D}$, with emphasis on improving data quality and cross-country comparability. ${ }^{49}$ The standard protocol includes digital representation of visual aids to assist the respondent throughout the interview (see figures 2 and 3). The study will use the portable version of the software, EQ-PVT, which for the respondent has the same functionality and for the most part resembles the standard EQ-VT software package.

The interview will start with administration of the EQ-5D-5L questionnaire, including the Visual Analogue Scale (VAS), followed by background questions for age, sex and experience with serious illness. Next, cTTO is administered, beginning with an explanation of the task demonstrated with 'the wheelchair example' including an introduction to the 'worse than dead' part of the task.

\begin{tabular}{lllll}
\hline \multicolumn{2}{l}{ Table 2} & \multicolumn{2}{l}{ Example sampling of hospital catchment areas and quotas per catchment area } & \\
\hline Region & Population in region & Catchment area & Population in catchment area & Quota per catchment area \\
\hline Northern & 381907 & Hospital 1 & 130000 & 140 \\
Central & 560690 & Hospital 2 & 60000 & 205 \\
Western & 843899 & Hospital 3 & 330000 & 309 \\
South-Eastern & 2299890 & Hospital 4 & 500000 & 448 \\
South-Eastern & $"$ & Hospital 5 & 160000 & 143 \\
South-Eastern & $"$ & Hospital 6 & 280000 & 251 \\
\hline
\end{tabular}


Table 3 Example of quotas within a sampled catchment area based on the composition of sex, age and educational level in the general population of the respective region (source: official statistics for 2017 generated from microdata.no)

\begin{tabular}{|c|c|c|c|c|c|c|c|c|c|}
\hline \multirow[b]{2}{*}{ Sex } & \multirow{2}{*}{$\begin{array}{l}\text { Highest attained } \\
\text { educational level }\end{array}$} & \multicolumn{7}{|c|}{ Age groups } & \multirow{2}{*}{$\begin{array}{l}\text { Total quota per sex } \\
\text { and educational leve }\end{array}$} \\
\hline & & $18-24$ & 25-34 & $35-44$ & $45-54$ & $55-64$ & $65-74$ & $75+$ & \\
\hline Male & Primary or secondary & 8 & 9 & 8 & 9 & 9 & 7 & 5 & 56 \\
\hline \multirow[t]{2}{*}{ Female } & Primary or secondary & 7 & 6 & 5 & 7 & 8 & 8 & 7 & 47 \\
\hline & Tertiary & 1 & 5 & 5 & 5 & 3 & 2 & 1 & 22 \\
\hline
\end{tabular}

Example given sampling scenario and catchment area for Hospital 1 in table 2.

This is followed by practice tasks for three states described with the EQ-5D-5L descriptive system, selected to reflect a mild, moderate and severe health state, familiarising the respondent further with the cTTO, the concept of health states worse than being dead and the use of lead-time in the cTTO for the valuation of such states. Lastly, respondents are administered their current health state as a cTTO task, allowing for the comparison of how respondents value their own health state with both cTTO and VAS.

Respondents are randomised to 1 of 10 TTO blocks of EQ-5D-5L health states, each consisting of 10 health states, one of which is always the worst state (level 5 on each dimension, state 55555), and one among the 5 mildest states $(11112,11121,11211,12111$ and 21111), for a total of 86 unique EQ-5D-5L health states for direct valuation. ${ }^{49}$ Respondents get the opportunity to review their responses in a feedback module (see figure 4), where individual task responses can be removed. On completion of the TTO tasks, respondents are randomised to 1 of 28 state pair blocks for discrete choices, each block consisting of seven state pairs. In both the TTO and DCE parts of the interview, the order of presentation is randomised. The randomised TTO and DCE tasks do not explicitly include a valuation of the respondents own health state, however, respondents can by chance be presented their own health state as a choice, in which case the task will be completed as normal.

The interview ends with further background questions specific to this study relating to variables known to be associated with valuations of health states including caregiver status, educational level and marital status. ${ }^{56-59}$ The influence of such variables will be assessed for the final value set.

Table 4 Reference data for the calculation of quotas, data for 2018 (http://microdata.no, statistics Norway, data accessed: 12 March 2019)

\begin{tabular}{|c|c|c|c|c|c|c|c|c|c|}
\hline \multirow[b]{2}{*}{ Region } & \multirow[b]{2}{*}{ Sex } & \multirow{2}{*}{$\begin{array}{l}\text { Highest attained } \\
\text { educational level }\end{array}$} & \multicolumn{7}{|c|}{ Age group } \\
\hline & & & $18-24$ & $25-34$ & $35-44$ & $45-54$ & $55-64$ & $65-74$ & $75+$ \\
\hline \multirow{4}{*}{$\begin{array}{l}\text { South- } \\
\text { Eastern } \\
\text { region }\end{array}$} & Male & Primary or secondary & 117220 & 130448 & 133470 & 143252 & 119278 & 94473 & 62167 \\
\hline & & Tertiary & 13603 & 72661 & 77273 & 66785 & 51553 & 40368 & 19650 \\
\hline & Female & Primary or secondary & 100571 & 94904 & 99033 & 120226 & 114228 & 107739 & 103859 \\
\hline & & Tertiary & 24196 & 104395 & 101833 & 79908 & 55565 & 34126 & 17126 \\
\hline \multirow{4}{*}{$\begin{array}{l}\text { Western } \\
\text { region }\end{array}$} & Male & Primary or secondary & 48863 & 54616 & 52141 & 54172 & 44925 & 34032 & 23977 \\
\hline & & Tertiary & 5129 & 26041 & 27176 & 21446 & 17552 & 12302 & 5291 \\
\hline & Female & Primary or secondary & 40743 & 35932 & 34778 & 42701 & 40672 & 36866 & 38127 \\
\hline & & Tertiary & 9928 & 39550 & 36796 & 27107 & 18494 & 9777 & 4750 \\
\hline \multirow{4}{*}{$\begin{array}{l}\text { Central } \\
\text { region }\end{array}$} & Male & Primary or secondary & 32425 & 33771 & 32095 & 36110 & 32525 & 26289 & 18441 \\
\hline & & Tertiary & 3674 & 15730 & 15703 & 13497 & 11291 & 8664 & 3521 \\
\hline & Female & Primary or secondary & 26707 & 21526 & 21130 & 28292 & 29998 & 28218 & 28275 \\
\hline & & Tertiary & 6456 & 23177 & 22577 & 18320 & 12267 & 6980 & 3024 \\
\hline \multirow{4}{*}{$\begin{array}{l}\text { Northern } \\
\text { region }\end{array}$} & Male & Primary or secondary & 22976 & 23320 & 21793 & 25812 & 23582 & 20282 & 13464 \\
\hline & & Tertiary & 1736 & 7895 & 8724 & 9427 & 7450 & 5273 & 1845 \\
\hline & Female & Primary or secondary & 18357 & 15382 & 14562 & 19478 & 20589 & 20492 & 19767 \\
\hline & & Tertiary & 3470 & 13212 & 14402 & 13721 & 8872 & 4300 & 1707 \\
\hline
\end{tabular}


Which is better, life A, life B, or are they about the same?

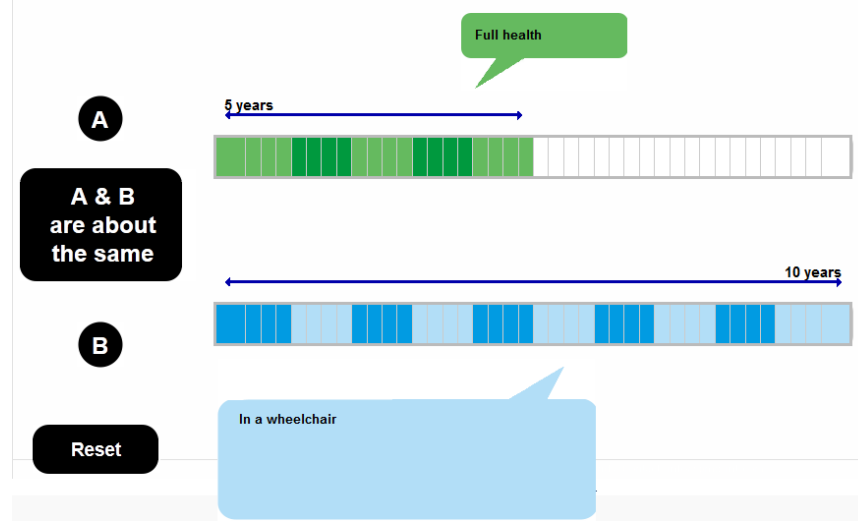

Figure 2 Screenshot of visual aid for cTTO task in EQ-VT. cTTO, composite time trade-off; EQ-VT, EuroQol valuation technology.

\section{Analysis}

The demographic characteristics and health status, that is, EQ-5D-5L profile, of respondents will be assessed and compared with national data. Parallel to this study, the NIPH has initiated data collection for a postal survey assessing the health status of the Norwegian population using the EQ-5D-5L, allowing for comparison of the health status of study populations. Health state values for EQ-5D-5L will be estimated through statistical modelling of the survey data. The EQ-5D protocols are not prescriptive with regard to modelling and approaches will depend on the characteristics of the data obtained. ${ }^{50}$ Following previous research, different models will be assessed including either the cTTO data, or combining the cTTO and DCE data in a hybrid model, and the results compared for adequacy with those for existing national value sets. $^{14-25}$ Modelling of values for the national value set will exclude valuations from respondents recruited from locations specifically for the collection of experience-based values and the valuations of respondents' own health state. Subgroup analysis will identify variables contributing to health state valuation in the Norwegian population. Values for health states defined as respondents' 'own health today' will be compared with values estimated for the same health states by the general population. In addition, all experienced-based valuations by those with serious illness and/or less than perfect health will be compared with valuations based on the total general population sample and, given sufficient data, those without experience of serious illness and/ or with perfect health today. To assess experience-based valuations, and explore both the wider and more narrow concepts of experience-based valuations, ${ }^{48}$ three potential profiles will be assessed: (1) respondents' valuation of own health state, (2) valuations given by respondents recruited from locations specifically chosen to target those with poorer health, that is, health services, (3) valuations given by respondents who have indicated that they have experience with serious illness.

\section{Patient and public involvement}

Patients and members of the public were not invited to comment on the study design or contribute to the writing or editing of this document for readability or accuracy.

\section{Strengths and limitations}

This is the first Norwegian valuation study with both cTTO and DCE undertaken on a scale large enough to meet the most recent EQ-5D protocol. The study intends to complete 1300-1500 face-to-face computer-assisted interviews across a country with a relatively dispersed population of citizens and large geographical distances
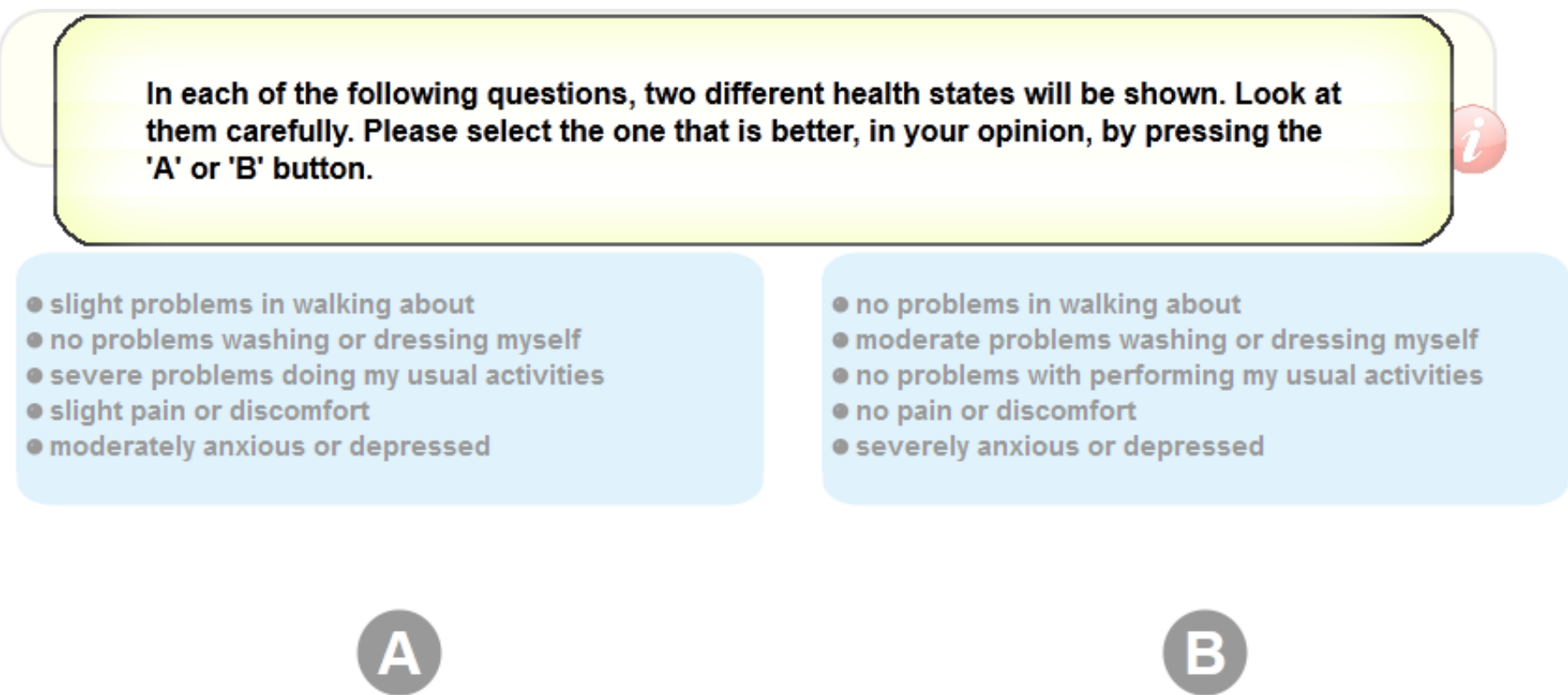

Figure 3 Screenshot of visual aid for DCE task in EQ-VT. DCE, discrete choice experiments; EQ-VT, EuroQol valuation technology. 


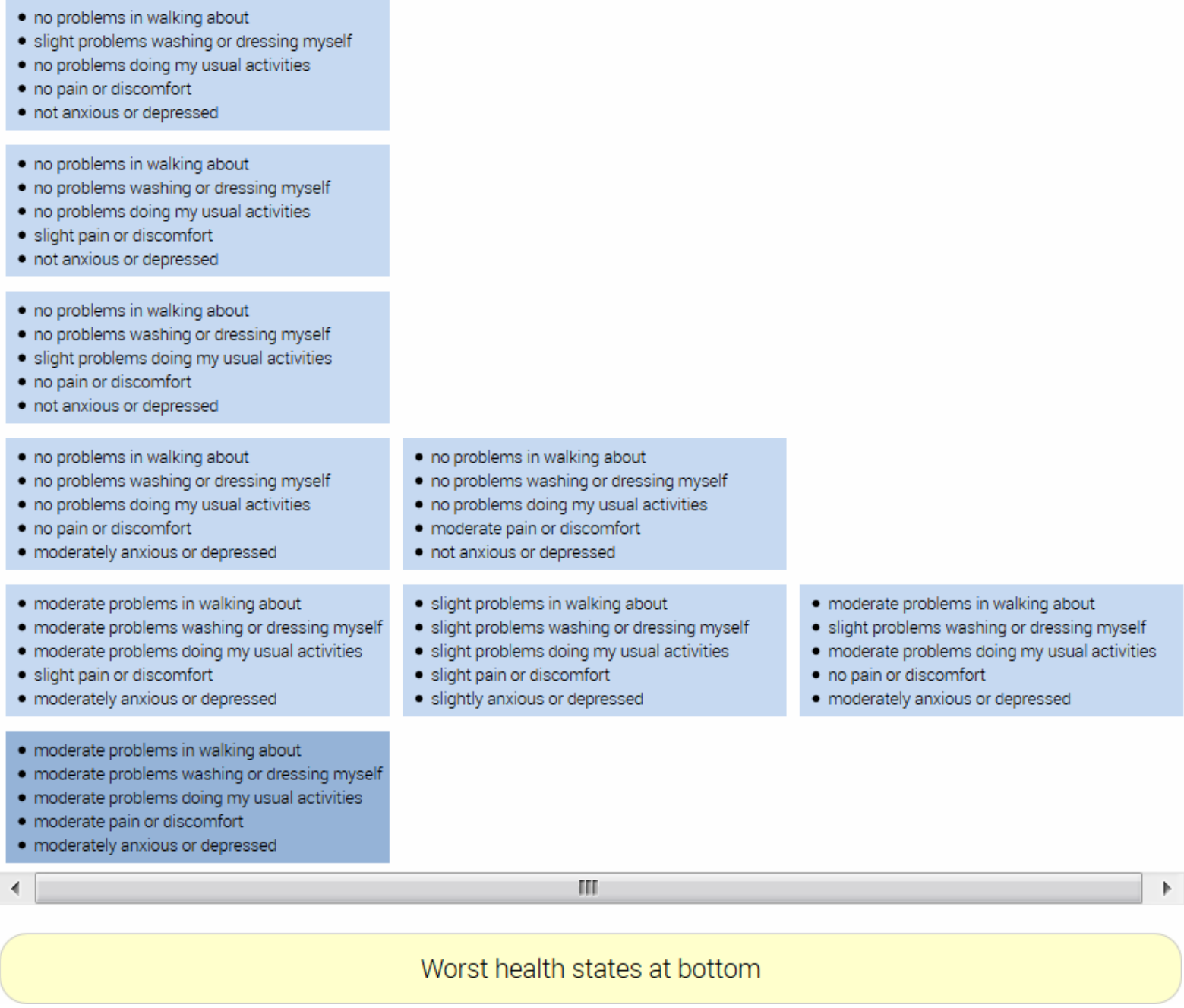

Figure 4 Screenshot of the feedback module in EQ-VT. EQ-VT, EuroQol valuation technology.

between them. Data collection involves a small number of interviewers working over an 8-month period.

Both the duration and magnitude of the tasks involved make the interview demanding. It is important that data collection is cost-effective, which includes considerations of data quality, representativeness and total number of valuations. Given the strategy of sampling locations and organisations rather than individuals, the assessment of its effectiveness in terms of number and representativeness of respondents will be important following the initial data collection period. Poor recruitment and data collection in remote geographical locations will be costly. The number and characteristics of respondents per location will be monitored throughout data collection. Adaptive sampling will allow for inclusion of additional locations where response rates are low and quotas are not met. Additional locations will be chosen at random from the predefined frame of possible locations within the selected geographical area.
Due to the need for extensive training, interview experience and understanding of the task, only 8-12 interviewers will be included. This will give more control over the data collection and the quality of the data collected. However, this and potential costs saved in terms of interviewer recruitment, training and travel costs, must be balanced against the increased impact of any loss of interviewers through illness or resignation during data collection. Norway has a harsh winter climate and apart from the Southern and Eastern region, where the interviewers are based, the interviews will primarily take place outside the winter months to reduce the risk of travel delays and interviewer illness. The NIPH, which is conducting the research, has several experienced interviewers familiar with the study who will be able to complete training and contribute to data collection if needed.

The main justification for the strategy of sampling stratified locations and the use of quotas on the respondent level is to ensure representativeness of the final sample 
according to geographical region, age, sex and educational level. An additional quota will be used to recruit those with less than perfect health, through locations such as hospitals and rehabilitation centres. Locations will also be selected to directly seek out others who are typically harder to reach and are less likely to participate in research studies, such as those with different ethnic backgrounds or with young children. Studies have found that some attributes, such as marital and caregiver status/ having young children, may influence the respondents response to the task, such as their willingness to trade time in the TTO task, despite showing similar preferences for given health states when using other types of valuation tasks. ${ }^{56}{ }^{58}$ Hence, it is important that respondents with such attributes are included in the study and locations such as day care facilities for young children and primary schools will be selected to facilitate this. Questions relating to these attributes will be included in the background questions closing the interview, and as such will allow for subgroup analysis of the effect of these attributes on the valuation of health in the Norwegian sample.

The derivation of values based on experienced health states is a recent development in the field of health state valuation. ${ }^{48}$ In recent years, there have been major developments in the field of standardised protocols for health state valuation, including EQ-VT. Such standardisation is a long way off for experienced health state valuation and, as was the case for hypothetical health state valuation up until the last decade, there is considerable variation in the choice of methods. ${ }^{60}$ In Norway and other countries, the feasibility of collecting such data is still in its infancy, including choice of sampling strategies, recruitment and how to minimise respondent burden. This study builds on existing methodology in the form of EQ-VT protocol, to assess the feasibility of recruiting potential respondents (including from healthcare settings) for experiencebased health state valuation, respondent burden in the form of completed interviews and data quality. The study design is constrained by the EQ-VT protocol, but the results of the study will inform the development of more appropriate methodology in the future. Furthermore, the design will allow the comparison of results with those for hypothetical health state valuation.

\section{ETHICS AND DISSEMINATION}

The study was reviewed by The Regional Health Authority Research Ethics Committee and found to be outside of the scope of the ethics committee thus not in need of ethical approval. All study participants will give informed consent.

The final scoring algorithm will contribute to the quality and relevance of the results of EQ-5D applications in Norway, and it is highly likely that, when available, the EQ-5D-5L with a Norwegian scoring algorithm will be the recommended instrument of choice for future economic evaluations undertaken in Norway by the pharmaceutical industry and other important users. Application of the same instrument and scoring across the health services and industry will further enhance decision-making relating to scarce healthcare resources. Moreover, scores based on Norwegian preferences will further enhance the appropriateness of the EQ-5D in clinical and health services research and quality indicators work, including the national quality registers.

The study results will be published in peer-review scientific journals, presented at appropriate forums, including national and international conferences, and scoring algorithms made publicly available for R, Stata and other widely used statistical software. Presentations will be given to users of the research, including research centres that widely use the EQ-5D in clinical, health services and health economics research in Norway.

Contributors AG conceived the study and secured funding. TMH, YH and AG designed the study. LAA, KR and KS commented and recommended revisions. TMH and $A G$ drafted and revised the manuscript. YH, LAA, KR and KS have read and approved the final version. All authors agree to be accountable for all aspects of the work.

Funding The study is funded by the Norwegian Research Council (grant number: 262673) with additional support from the EuroQol Foundation (EQ Project 20190280) and Norwegian Institute of Public Health.

Map disclaimer The depiction of boundaries on this map does not imply the expression of any opinion whatsoever on the part of BMJ (or any member of its group) concerning the legal status of any country, territory, jurisdiction or area or of its authorities. This map is provided without any warranty of any kind, either express or implied.

Competing interests None declared.

Patient consent for publication Not required.

Provenance and peer review Not commissioned; externally peer reviewed.

Open access This is an open access article distributed in accordance with the Creative Commons Attribution Non Commercial (CC BY-NC 4.0) license, which permits others to distribute, remix, adapt, build upon this work non-commercially, and license their derivative works on different terms, provided the original work is properly cited, appropriate credit is given, any changes made indicated, and the use is non-commercial. See: http://creativecommons.org/licenses/by-nc/4.0/.

ORCID iD

Tonya Moen Hansen http://orcid.org/0000-0003-3150-4765

\section{REFERENCES}

1 Hagen G, Wisløff T, Klemp M. Helseøkonomisk evaluering ved Nasjonalt kunnskapssenter for helsetjenesten. Nor Epidemiol 2013;23:157-64.

2 Statens Legemiddelverk (National Medicine Agency). Guidelines for the submission of documentation for single technology assessment (STA) of pharmaceuticals, 2018.

3 Ottersen T, Førde R, Kakad M, et al. A new proposal for priority setting in Norway: open and fair. Health Policy 2016;120:246-51.

4 På ramme alvor: alvorlighet og prioritering. Report from Working group appointed by Norwegian Ministry of health and care services; 2015.

5 Norwegian Ministry of Health and Care Services. Principles for priority setting in health care- summary of a white paper on priority setting in the Norwegian health care sector; 2015.

6 Drummond MF SM, Torrance G, O'Brien B, et al. Methods for the economic evaluation of health care programmes. 3rd edn. Oxford University Press, 2005.

7 Devlin NJ, Brooks R. EQ-5D and the EuroQol group: past, present and future. Appl Health Econ Health Policy 2017;15:127-37.

8 Garratt A, Schmidt L, Mackintosh A, et al. Quality of life measurement: bibliographic study of patient assessed health outcome measures. BMJ 2002;324:1417. 
9 Nord E. Cost-Value analysis in health care: making sense out of QALYS. Cambridge University Press, 1999.

10 Wisløff T, Hagen G, Hamidi V, et al. Estimating QALY gains in applied studies: a review of cost-utility analyses published in 2010. Pharmacoeconomics 2014;32:367-75.

11 EuroQol. EQ-5D-5L | Self-complete version on paper. Available: https://euroqol.org/eq-5d-instruments/eq-5d-5l-available-modes-ofadministration/self-complete-on-paper/

12 EuroQol. Sample UK English EQ-5D-5L paper Self-complete version.

13 Szende A OM, Devlin N, eds. EQ-5D Value Sets: Inventory, Comparative Review and User Guide. Dordrecht, The Netherlands: Springer, 2007.

14 Devlin NJ, Shah KK, Feng Y, et al. Valuing health-related quality of life: an EQ-5D-5L value set for England. Health Econ 2018;27:7-22.

15 Ludwig K, Graf von der Schulenburg J-M, Greiner W. German value set for the EQ-5D-5L. Pharmacoeconomics 2018;36:663-74.

16 Augustovski F, Rey-Ares L, Irazola V, et al. An EQ-5D-5L value set based on Uruguayan population preferences. Qual Life Res 2016;25:323-33.

17 Hobbins A, Barry L, Kelleher D, et al. Utility values for health states in Ireland: a value set for the EQ-5D-5L. Pharmacoeconomics 2018;36:1345-53.

18 Kim S-H, Ahn J, Ock M, et al. The EQ-5D-5L valuation study in Korea. Qual Life Res 2016;25:1845-52.

19 Luo N, Liu G, Li M, et al. Estimating an EQ-5D-5L value set for China. Value Health 2017:20:662-9.

20 M Versteegh M, M Vermeulen K, M A A Evers S, et al. Dutch tariff for the five-level version of EQ-5D. Value Health 2016;19:343-52.

21 Pattanaphesaj J, Thavorncharoensap M, Ramos-Goñi JM, et al. The EQ-5D-5L valuation study in Thailand. Expert Rev Pharmacoecon Outcomes Res 2018:18:551-8.

22 Purba FD, Hunfeld JAM, Iskandarsyah A, et al. The Indonesian EQ5D-5L value set. Pharmacoeconomics 2017;35:1153-65.

23 Shafie AA, Vasan Thakumar A, Lim CJ, et al. EQ-5D-5L valuation for the Malaysian population. Pharmacoeconomics 2019;37:715-25.

24 Wong ELY, Ramos-Goñi JM, Cheung AWL, et al. Assessing the use of a feedback module to model EQ-5D-5L health states values in Hong Kong. Patient 2018;11:235-47.

25 Xie F, Pullenayegum E, Gaebel K, et al. A time Trade-off-derived value set of the EQ-5D-5L for Canada. Med Care 2016;54:98-105.

26 Solberg TK, Olsen J-A, Ingebrigtsen T, et al. Health-related quality of life assessment by the EuroQol-5D can provide cost-utility data in the field of low-back surgery. Eur Spine J 2005;14:1000-7.

27 Nilsson E, Orwelius L, Kristenson M. Patient-reported outcomes in the Swedish national quality registers. J Intern Med 2016;279:141-53.

28 Hernández-Alava M, Pudney S, Wailoo A. Quality review of a proposed EQ-5D-5L value set for England. Policy Research Unit in Economic Evaluation of Health and Care Interventions. Universities of Sheffield and York: EEPRU Research, 2018.

29 National Institute for Health and Care Excellence. Position statement on use of the EQ-5D-5L valuation set for England (updated November 2019), 2019. Available: https://www.nice.org.uk/about/ what-we-do/our-programmes/nice-guidance/technology-appraisalguidance/eq- $5 d-5 l$

30 Dolan P. Modeling valuations for EuroQol health states. Med Care 1997;35:1095-108.

31 van Hout B, Janssen MF, Feng Y-S, et al. Interim scoring for the EQ$5 \mathrm{D}-5 \mathrm{~L}$ : mapping the EQ-5D-5L to EQ-5D-3L value sets. Value Health 2012;15:708-15.

32 Kularatna S, Chen G, Byrnes J, et al. Mapping Sri Lankan EQ-5D-3L to EQ-5D-5L value sets. Value Health Reg Issues 2017;12:20-3.

33 Golicki D, Niewada M, Hout Bvan, et al. Interim EQ-5D-5L value set for Poland: first Crosswalk value set in central and eastern Europe. Value Health Reg Issues 2014;4:19-23.

34 Olsen JA, Lamu AN, Cairns J. In search of a common currency: a comparison of seven EQ-5D-5L value sets. Health Econ 2018;27:39-49.
35 Bailey $\mathrm{H}$, Kind P. Preliminary findings of an investigation into the relationship between national culture and $\mathrm{EQ}-5 \mathrm{D}$ value sets. Qual Life Res 2010;19:1145-54.

36 Burström K, Sun S, Gerdtham U-G, et al. Swedish experience-based value sets for EQ-5D health states. Qual Life Res 2014;23:431-42.

37 Leidl R, Reitmeir P. An experience-based value set for the EQ-5D-5L in Germany. Value Health 2017;20:1150-6.

38 Sun S, Chen J, Kind P, et al. Experience-based VAS values for EQ$5 \mathrm{D}-3 \mathrm{~L}$ health states in a national general population health survey in China. Qual Life Res 2015;24:693-703.

39 NICE. Guide to the methods of technology appraisal. London: National Institute for Health and Clinical Excellence, 2008.

40 Brazier J, Akehurst R, Brennan A, et al. Should patients have a greater role in valuing health states? Appl Health Econ Health Policy 2005;4:201-8.

41 Dolan P. Thinking about it: thoughts about health and valuing QALYs. Health Econ 2011;20:1407-16.

42 TLV. Stivarga kommer inte att ingå i högkostnadsskyddet. [Stivarga will not be reimbursed]. Stockholm: TLV, 2014.

43 Leidl R, Reitmeir P. A value set for the EQ-5D based on experienced health states: development and testing for the German population. Pharmacoeconomics 2011;29:521-34.

44 De Wit GA, Busschbach JJ, De Charro FT. Sensitivity and perspective in the valuation of health status: whose values count? Health Econ 2000;9:109-26.

45 Dolan P. NICE should value real experiences over hypothetical opinions. Nature 2009;462:35.

46 Heijink R, Reitmeir P, Leidl R. International comparison of experience-based health state values at the population level. Health Qual Life Outcomes 2017;15:138.

47 Dolan P, Kahneman D. Interpretations of utility and their implications for the valuation of health. Econ $J$ 2008;118:215-34.

48 Cubi-Molla P, Shah K, Burström K. Experience-Based values: a framework for classifying different types of experience in health valuation research. Patient 2018;11:253-70.

49 Oppe M, Devlin NJ, van Hout B, et al. A program of methodological research to arrive at the new international EQ-5D-5L valuation protocol. Value Health 2014:17:445-53.

50 Oppe M, Rand-Hendriksen K, Shah K, et al. EuroQol protocols for time trade-off valuation of health outcomes. Pharmacoeconomics 2016;34:993-1004

51 Galea S, Tracy M. Participation rates in epidemiologic studies. Ann Epidemiol 2007:17:643-53.

52 Microdata.no, 2019. Available: https://microdata.no/en/

53 Purba FD, Hunfeld JAM, Iskandarsyah A, et al. Employing quality control and feedback to the EQ-5D-5L valuation protocol to improve the quality of data collection. Qual Life Res 2017;26:1197-208.

54 Ramos-Goñi JM, Oppe M, Slaap B, et al. Quality control process for EQ-5D-5L valuation studies. Value Health 2017;20:466-73.

55 Stolk E, Ludwig K, Rand K, et al. Overview, update, and lessons learned from the International EQ-5D-5L valuation work: version 2 of the EQ-5D-5L valuation protocol. Value Health 2019;22:23-30.

56 van Nooten F, Busschbach J, van Agthoven M, et al. What should we know about the person behind a TTO? Eur J Health Econ 2018;19:1207-11.

57 Craig BM, Reeve BB, Cella D, et al. Demographic differences in health preferences in the United States. Med Care 2014:52:307-13.

58 van der Pol M, Shiell A. Extrinsic goals and time tradeoff. Med Decis Making 2007;27:406-13.

59 van Nooten FE, van Exel NJA, Koolman X, et al. "Married with children" the influence of significant others in TTO exercises. Health Qual Life Outcomes 2015;13:94.

60 Helgesson G, Ernstsson O, Åström M, et al. Whom should we ask? A systematic literature review of the arguments regarding the most accurate source of information for valuation of health states. Qual Life Res 2020;4. 